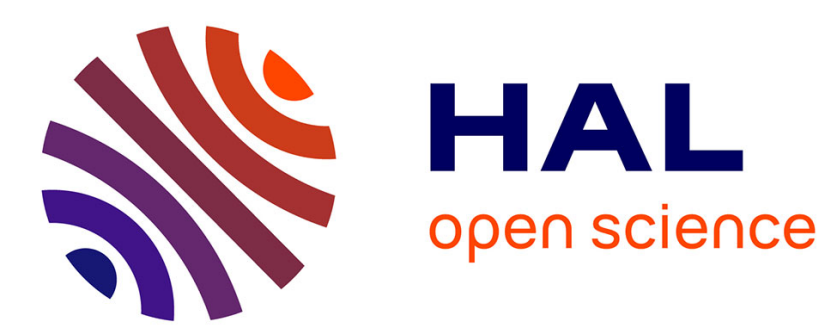

\title{
Wrapping-free numerical refocusing of scalar electromagnetic fields
}

Pascal Berto, Marc Guillon, Pierre Bon

\section{To cite this version:}

Pascal Berto, Marc Guillon, Pierre Bon. Wrapping-free numerical refocusing of scalar electromagnetic fields. Applied optics, 2018, 57 (22), pp.6582. 10.1364/AO.57.006582 . hal-02380410

\section{HAL Id: hal-02380410 https://hal.science/hal-02380410}

Submitted on 29 Nov 2019

HAL is a multi-disciplinary open access archive for the deposit and dissemination of scientific research documents, whether they are published or not. The documents may come from teaching and research institutions in France or abroad, or from public or private research centers.
L'archive ouverte pluridisciplinaire HAL, est destinée au dépôt et à la diffusion de documents scientifiques de niveau recherche, publiés ou non, émanant des établissements d'enseignement et de recherche français ou étrangers, des laboratoires publics ou privés. 


\title{
Wrapping-free numerical refocusing of scalar electromagnetic fields
}

\author{
Pascal Berto ${ }^{1}$, Marc Guillon ${ }^{1}$, and Pierre Bon ${ }^{*}, 2,3$ \\ ${ }^{1}$ Sorbonne Paris Cité, Université Paris Descartes, Neurophotonics Laboratory, CNRS-UMR 8250, 45 rue des Saints-Pères, F-75006 Paris, France \\ ${ }^{2}$ Univ. Bordeaux, Laboratoire Photonique Numérique et Nanosciences (LP2N), UMR 5298, F-33400 Talence, France \\ ${ }^{3}$ CNRS and Institut d'Optique, LP2N UMR 5298, F-33400 Talence, France. \\ ${ }^{*}$ Corresponding author: pierre.bon@u-bordeaux.fr \\ Compiled May 14, 2018
}

Numerical refocusing in any plane is one powerful feature granted by measuring both the amplitude and the phase of a coherent light beam. Here we introduce a method based on the first Rytov approximation of scalar electromagnetic fields which (i) allows numerical propagation without requiring phase-unwrapping after propagation and (ii) limits the effect of artificial phase singularities that appear upon numerical defocusing when the measurement-noise is mixing with the signal. We demonstrate the feasibility of this method with both scalar electromagnetic field simulations and real acquisitions of microscopic biological samples imaged at high numerical aperture.

(C) 2018 Optical Society of America

OCIS codes: 070.7345 Wave propagation, 090.1995 Digital holography, 110.7348 Wavefront encoding, 120.5050 Phase measurement, 180.3170 Interference microscopy

http://dx.doi.org/10.1364/ao.XX.XXXXXX

\section{INTRODUCTION}

Having the ability to measure not only the intensity but the scalar electromagnetic field (intensity and phase) is of high interest for many applications. In optical microscopy, phase information allows high-contrast imaging of biological semi-transparent samples. Complex amplitude measurement typically allows numerical focusing [1] which is very valuable for biological studies requiring hours or days of acquisition [2,3]. Tiny thermal instability (e.g. $\approx 1^{\mathrm{o}}$ variation) may indeed induce several microns of defocus [4].

Many different solutions have been proposed to retrieve the phase information. We may identify two categories: (i) those which directly retrieve the phase of the light by means of a reference beam (see e.g. [5-12]) - the most common technique being digital holography - and (ii) those which do not directly retreive the optical phase but rather a related quantity such as its transverse gradient map (see e.g. [13-19]) - the most common technique being wavefront sensing. In direct methods, phase is provided modulo $2 \pi$ : phase is then said to be "wrapped". Phase wrapping is intrinsically related to the phase quantity itself which is not a single valued physical quantity. Phase singularities resulting from so-called helical or edge phase "disclinations" can thus appear [20]. However, in microscopy applications, the phase quantity is usually related to the delay introduced by a thin sample, imprinting a smooth and regular distortion function to the wavefront. In this case, phase-delays larger than $2 \pi$ (commonly reached in real biological samples [5]) result in phase wrapping, irrelevant to the sought-for optical path length measurement. Phase unwrapping algorithms have then to be applied to retrieve a continuous phase profile [21]. Unwrapping can fail in the presence of noise - associated with accidental disclinations - or in the case of large phase gradients. The methods of the second phase-retrieval family are less prone to such artifacts because the measured quantity is not $2 \pi$-periodic. Wavefront sensor based methods [14-17] typically measure the wavefront derivatives rather than the phase map, so avoiding the wrapping problems.

However, numerical propagation of a complex wavefield typically requires computing the sine and cosine of the measured phase. The computed phase is thus wrapped, which is particularly regrettable when the imaged phase object is smooth and the original measured phase is retrieved in a wrapping-free manner.

In this work we propose a new algorithm based on the Rytov approximation $[22,23]$, working with unwrapped phase profiles. Propagation is thus computed on the phase itself, so without phase-map wrapping. It intrinsically avoids the generation of phase singularities when noise is mixed with the signal to be propagated. At first order approximation, for small defocus, the Rytov field propagation provides the same result as EM field propagation while removing the requirement for phase unwrapping after propagation. We also demonstrate that large defocus ranges can also be computed in a step-by-step manner, by updating the amplitude term at every step.

\section{PUPIL FUNCTION AND CLASSICAL APPROACH FOR NUMERICAL REFOCUSING}

When considering quantitative phase and intensity imaging, it is convenient to consider the image formation in the Fourier 
space. For a plane wave illumination with wave-vector $\overrightarrow{k_{0}}$, the diffracted wavevector $\overrightarrow{k_{d}}$ must satisfy:

$$
\left\{\begin{array}{l}
\overrightarrow{k_{d}}=\overrightarrow{k_{0}}+\vec{K} \\
\left\|\overrightarrow{k_{d}}\right\|=\left\|\overrightarrow{k_{0}}\right\|
\end{array},\right.
$$

with $k_{0}=2 \pi / \lambda, \lambda$ the illumination wavelength in the immersion medium of the objective, and $K$ the object spatial frequencies. This equation system implies that the diffracted wavevectors are carried by the Ewald sphere[24, 25]. It is valid for propagating light-waves and under the single-scattering regime which is generally the case in phase imaging of thin semi-transparent samples.

Here, we consider that the sample is imaged in a transillumination scheme with a microscope objective of finite numerical aperture $N A$. With these assumptions, the exiting diffracted angles are carried by a portion of the Ewald sphere, implying a bijection between the transverse spatial frequencies of the object and the transverse wavevectors of the diffracted waves. Sampling of the scalar EM field in a single plane thus carries the full information for 3D wavefield reconstruction, so-allowing image refocusing. From equation 1 , the 3D point spread function (PSF) $H_{3 D}(x, y, z)$ of the microscope can be obtained via its Fourier Transform $\tilde{H}_{3 D}\left(K_{x}, K_{y}, K_{z}\right)$ :

$$
\begin{gathered}
\tilde{H}_{3 D}\left(K_{x}, K_{y}, K_{z}\right)=\delta\left(\left\|\overrightarrow{k_{d}}\right\|-\left\|\overrightarrow{k_{0}}\right\|\right) \text { if } N A \cdot k_{0} \geq \rho \\
=0 \quad \text { else, }
\end{gathered}
$$

with $\delta$ the Dirac function and $\rho=\sqrt{k_{d, x}^{2}+k_{d, y}^{2}}$. In the scope of numerical refocusing in one plane, it is interesting to consider one slice of this PSF in the Fourier space. For simplification, let us consider an illumination along the optical axis (i.e. $\overrightarrow{k_{0}}=$ $k_{0} \cdot \overrightarrow{u_{z}}$ ) as it is straightforward to generalize all the following formulas for a tilted illumination angle [26]. In this case $\rho=$ $\sqrt{k_{d, x}^{2}+k_{d, y}^{2}}=\sqrt{K_{x}^{2}+K_{y}^{2}}$ and we obtain from equation 1 and 2 :

$$
\begin{gathered}
\tilde{H}_{z=z_{0}}\left(K_{x}, K_{y}\right)=\mathrm{e}^{i \cdot k_{0} z_{0}\left[1-\sqrt{1-\frac{\rho^{2}}{k_{0}^{2}}}\right]} \text { if } N A \cdot k_{0} \geq \rho \\
=0 \quad \text { else, }
\end{gathered}
$$

with $z_{0}$ the distance to the best focus plane. Equation 3 is known as the pupil function for Fresnel diffraction theory.

Considering a scalar EM field $E_{z=0}(x, y)=A(x, y) \cdot e^{i \cdot \varphi(x, y)}$, one way to obtain a numerical refocusing in a plane $z=z_{0}$ is to convolve the EM field by the pupil function:

$$
E_{z=z_{0}}(x, y)=E_{z=0}(x, y) \otimes H_{z=z_{0}}(x, y)
$$

Which can be rewritten in the Fourier space as:

$$
\begin{aligned}
& \tilde{E}_{z=z_{0}}\left(K_{x}, K_{y}\right)=\tilde{E}_{z=0}\left(K_{x}, K_{y}\right) \cdot \mathrm{e}^{i \cdot k_{0} z_{0}\left[1-\sqrt{1-\frac{\rho^{2}}{k_{0}^{2}}}\right]} \text { if } N A \cdot k_{0} \geq \rho \\
& =0 \quad \text { else. }
\end{aligned}
$$

As previously mentioned, to perform the numerical propagation it is mandatory to work with the scalar EM field $E$. It means that the phase has to be introduced into the complex exponential before propagation. Thus, the propagated phase will also be wrapped between 0 and $2 \pi$.

\section{FIRST RYTOV APPROXIMATION AND STEP-BY-STEP NUMERICAL PROPAGATION}

To allow a wrapping free numerical propagation, we propose not to use directly the scalar EM field but rather its first order Rytov approximation. The first Rytov approximation has been widely used in diffraction tomography to retrieve $3 \mathrm{D}$ refractive index distribution from phase and intensity measurements [22, 25]. We define the Rytov EM field $E_{\text {Rytov }}$ as:

$$
E_{\text {Rytov }}(x, y)=\ln (E(x, y))=\ln (A(x, y))+i \cdot \varphi(x, y) .
$$

The first Rytov approximation has been discussed to be valid when the spatial gradient of the field $\nabla E_{R y t o v}$ is smaller than the wavevector $k_{0}$ [27], which is commonly the case for biological samples. Numerical propagation of $E_{\text {Rytov }}$ can then be achieved using the pupil function $H$ defined in equation 3 :

$$
E_{R y t o v, z=z_{0}}(x, y)=E_{R y t o v, z=0}(x, y) \otimes H_{z=z_{0}}(x, y) .
$$

Let us now discuss more quantitatively the accuracy of the Rytov field approximation for numerical refocusing compared to conventional numerical refocusing using the scalar EM field $E$. Because the calculation is done in the Fourier space, we propose to evaluate the performance of the Rytov field propagation versus the EM field propagation by considering single-frequency phase modulation signals $\varphi(x, y)=\varphi_{0} \cdot \sin (2 \pi / P \cdot x)$, with $P$ the period of the phase modulation. According to the JacobiAnger expansion:

$$
e^{i \cdot \varphi_{0} \cdot \sin (\theta)}=\sum_{n=-\infty}^{+\infty} J_{n}\left(\varphi_{0}\right) e^{i \cdot n \cdot \theta},
$$

for large values of the phase modulation amplitude $\varphi_{0}$, the frequency support of the EM field is not a single frequency signal but harmonics are generated. This is not the case with the Rytov approximation and this is the main limitation of the approximation.

To quantify this assumption, we have compared the EM field propagation according to Eq. 5 and in the frame of the Rytov approximation (Eq. 7) by propagating single-frequency phase modulation signals (with uniform intensity illumination) with different amount of defocus, modulation amplitudes and phase modulation periods. Setting arbitrarily a maximum tolerable discrepancy of $5 \%$ (in standard deviation) between approximated and exact propagated phase, we obtained the figure 1.a. It shows a chart of the corresponding maximum modulation amplitude as a function of defocus and phase modulation periods. These simulation results have been obtained using $\lambda=500 \mathrm{~nm}$ and $N A=1.3$. Phase modulation periods are shown in log scale both in $\mu \mathrm{m}$ and in fraction of the coherent point spread radius $r_{P S F}$. The defocus is also in log-scale both in $\mu \mathrm{m}$ and in Rayleigh distance $d_{\text {Rayleigh }}$. Noteworthy, the high frequencies are more affected by the Rytov approximation while the lower frequencies tend to be conserved even with very high defocus and phase modulation. For example, for spatial scales comparable with large biological mammal cells (i.e. period of $\approx 30 \mu \mathrm{m}$ ) the Rytov approximation induces less than $5 \%$ of differences even with $10 \lambda$ of phase modulation and $100 \mu \mathrm{m}$ defocus. This amount is very large compared to the observable conventional values of $\approx 1 \lambda$ of phase shift for a regular cell [14, 17]. 
To minimize errors obtained on the phase when propagating the Rytov field over long distances, we suggest a step-by-step refocusing algorithm. Let us describe the motivation of this algorithm. The propagated intensity using the Rytov approximation or the regular EM field are also exhibiting differences. Concurrently, when using the EM field propagation, the intensity $I_{E M}$ accumulates more and more signatures from the phase while being preserved from any wrapping or singularity error. It is then interesting to take into account $I_{E M}$ while using the Rytov propagation. It improves the reliability while preserving the advantages associated with phase calculation by Rytov propagation. We have thus built a step-by-step propagation algorithm and update the pristine intensity $I_{E M}$ at every step, all along the propagation. At step $m$, the intensity $I_{E M}((m-1) \delta)$ obtained by propagating the EM field (Eq. 5) and the phase $\varphi_{\text {Rytov }}((m-1) \delta)$ obtained by propagating the Rytov field (Eq. 7) are combined using Eq. 6 and then propagated over a distance $\delta$ using Eq. 7. The algorithm flow-chart is presented in fig. 2.

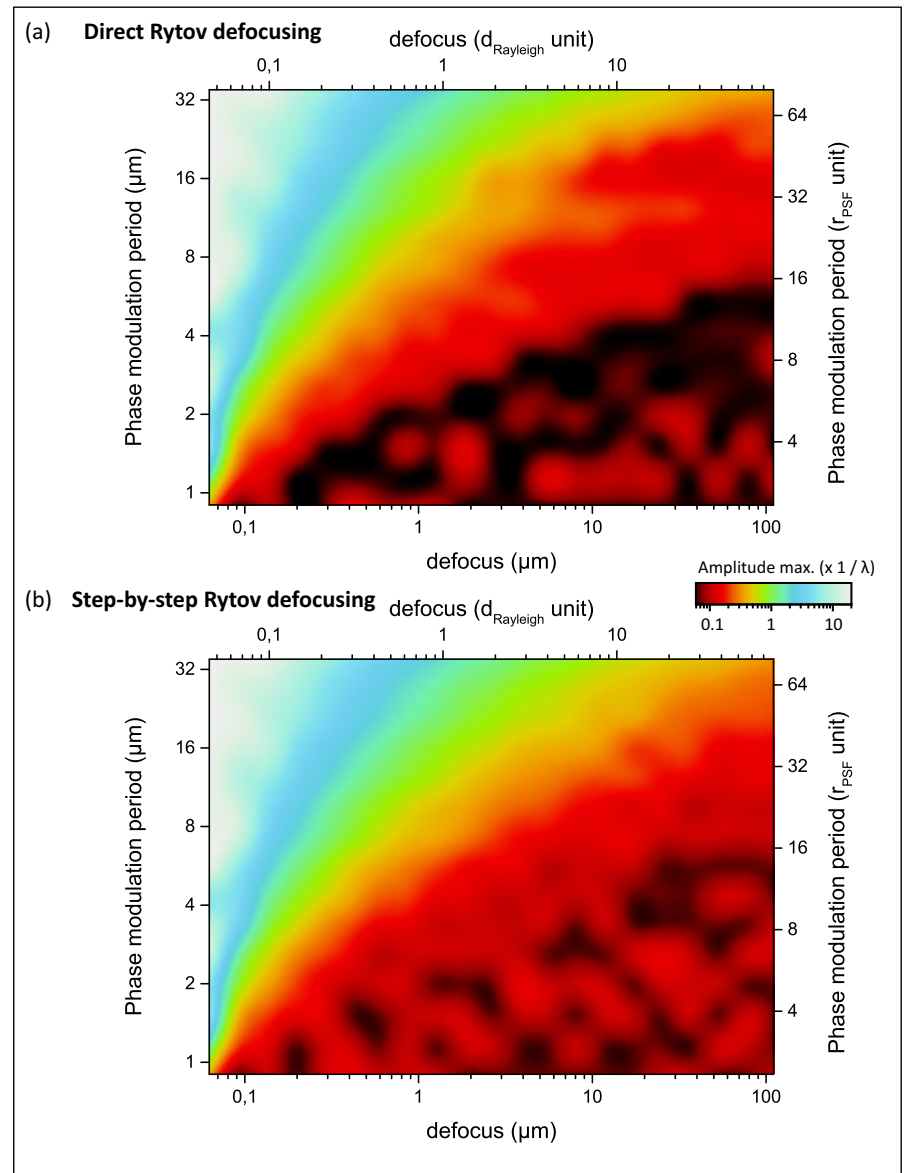

Fig. 1. Validity domain of the Rytov approximation for numerical propagation. Comparison between direct and stepby-step propagation (a) Maximum amplitude for pure-sine phase modulation leading to less than $5 \%$ error between a direct propagation with the Rytov approximation and conventional scalar EM field propagation. (b) Same as (a) with a step-by-step Rytov approximation propagation

This algorithm is more time consuming than direct Rytov and EM field numerical refocusing. It requires to perform $3 \times N+1$ Fourier transforms with $N$ the number of iterations of the algorithm while direct refocusing requires only 2 Fourier transforms. However the accuracy is much improved. As for direct Rytov field refocusing, we have studied the discrepancy between pristine EM field propagation and step-by-step Rytov field propagation of single-frequency phase modulation signals (uniform intensity illumination) for different amount of defocus, modulation amplitudes and phase modulation periods. Considering the same $5 \%$-error tolerance as for Fig. 1.a, chart 1.b was computed. In this step-by-step algorithm, the result accuracy depends on the step magnitude $\delta$. However, we observed that results did not change significantly when considering steps $\delta \leq \lambda / 2$. The simulation results have thus been obtained taking $\delta=\lambda / 2$ (and using the same parameters as for Fig. 1.a: $\lambda=500$ $\mathrm{nm}, N A=1.3)$. As expected, for large defocus $\left(>8 d_{\text {Rayleigh }}\right)$ step-by-step Rytov propagation is more accurate than direct numerical refocusing using the Rytov approximation. Moreover, for high-frequency phase-modulations, the reconstruction is possible with higher phase-modulation values (from 2 to $10 \times$ more) using the Rytov step-by-step algorithm. This means that the diffraction rings and the small details in the images will be preserved.

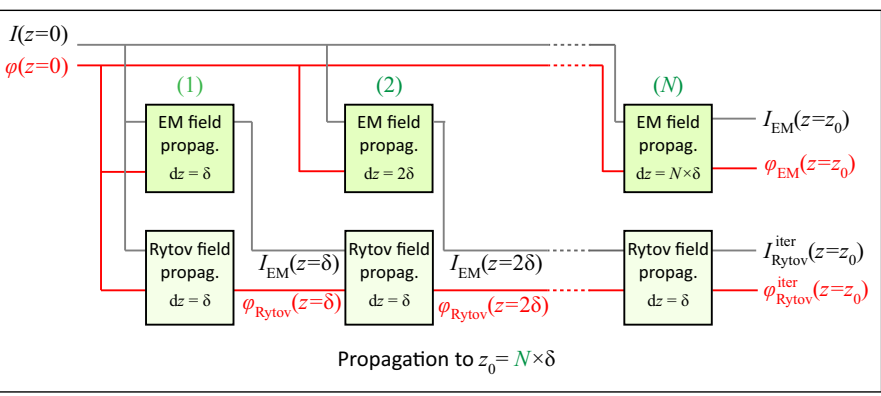

Fig. 2. Step-by-step propagation algorithm using the first Rytov approximation. At every step, the intensity is updated by propagating the EM field.

\section{EXPERIMENTAL VALIDATION}

Let us now discuss the potential of the Rytov approximation for numerical propagation of real phase measurements. First we considered a sample composed of a $5 \mu \mathrm{m}$ polystyrene bead immersed in an immersion oil (Nikon, USA) of refractive index 1.51. The refractive index mismatch is thus $\Delta n=0.07$ and the bead maximum optical path difference is $350 \mathrm{~nm}$. This sample can be considered as a phantom of a small suspended living cell. In figure 3.a a typical in-focus phase image is presented; this transmission-image is obtain using a commercial quadriwave lateral shearing interferometer (SID4Bio, Phasics) [14] plugged on a conventional microscope (Nikon TiE) equipped with $100 \times$ $\mathrm{NA}=1.3$ objective. The sample is illuminated by a halogen lamp with a bandwidth limited to $700 \pm 50 \mathrm{~nm}$ (Chroma). The spatial phase noise is negligible $\left(\sigma_{\text {noise }}=0.3 \mathrm{~nm}\right)$ and the aim of this study is to discuss on experimental measurements the effect of the Rytov approximation for numerical propagation on a quasi noise-free image. Fig. 3.b shows the results of numerical refocusing using the EM field while fig. 3.c is obtained with Rytov field propagation.

Noteworthy, the difference between direct Rytov approximation refocusing and EM field numerical defocusing is negligible until $\approx 5 \mu \mathrm{m}$ of defocus. For step-by-step Rytov 
approximation defocusing, the error is negligible over $\approx 12$ $\mu \mathrm{m}$. This result is consistent with the theoretical study in fig. 1 for an object of principal period $7.6 r_{P S F}$, a phase modulation of $\lambda / 2$ and a propagation of $2.6 d_{\text {Rayleigh }}$ (direct refocusing) and $6.2 d_{\text {Rayleigh }}$ (step-by-step refocusing). Defocusing using direct Rytov approximation leads to phase images which are axially symmetrical respect to the best focus while EM field defocusing leads to non-symmetrical phase maps. Step-by-step Rytov propagation allows retrieving this asymmetric behavior. However, for regular refocusing near the image plane, the Rytov approximation is sufficient.

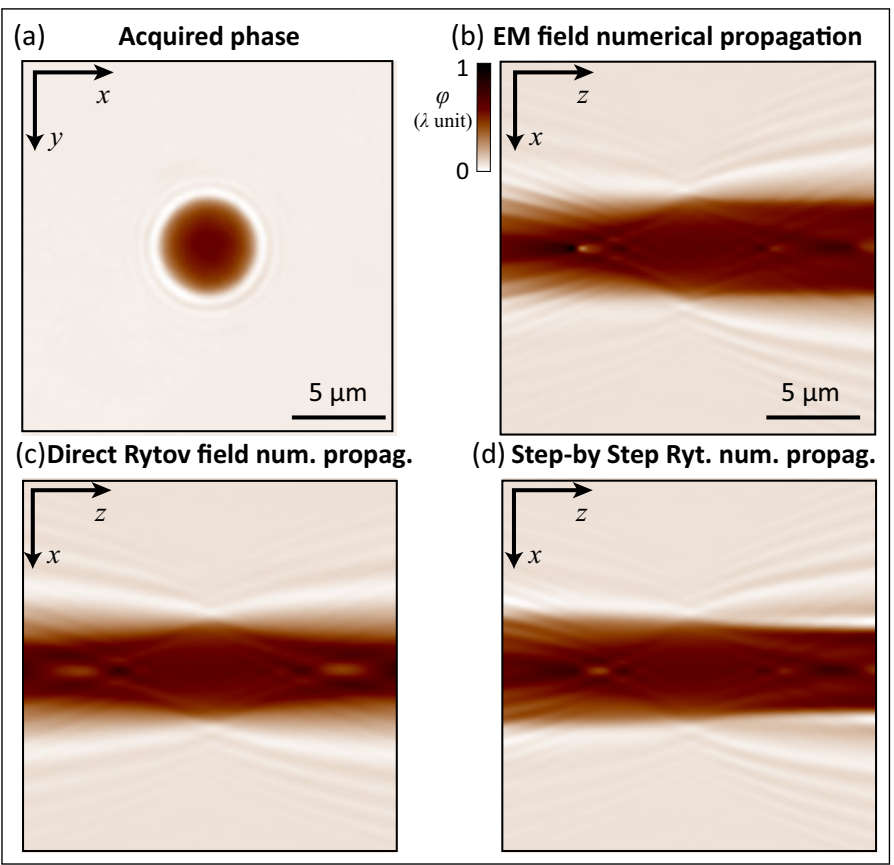

Fig. 3. Numerical defocusing on phantom (polystyrene bead). (a) Experimental quantitative phase image of a $5 \mu \mathrm{m}$ polystyrene bead in immersion oil with $\Delta n_{\text {polys./oil }}=0.07$. (b) Numerical defocusing using the EM field from $-10 \mu \mathrm{m}$ to $+10 \mu \mathrm{m}$. The image is a slice of the 3D phase stack along $y$ crossing the center of the bead. (c) Same as (b) with a numerical defocusing using the Rytov field. (d) Same as (b) with a step-by-step numerical propagation using the Rytov field.

Finally, we demonstrate the capability of our method to refocus phase images from biological samples inducing large phase-shifts. For this purpose, we have imaged a drosophila embryo using the same setup as presented before, the only difference being the wavelength adjusted to $510 \pm 25 \mathrm{~nm}$ (Semrock). A measured phase map is presented in fig. 4.a.

While the maximum phase shift is large (up to $7 \lambda$ ), the phase map is free from phase-wrapping since the quantitative phase sensor (quadriwave lateral shearing interferometer) is sentitive to the phase gradient and not the direct phase value. Numerical defocusing at $z=10 \mu \mathrm{m}$ using the EM field (Fig. 4.b), Rytov approximation with direct defocusing (Fig. 4.c) and Rytov approximation with step-by-step defocusing (Fig. 4.d) are considered. Even with unwrapping algorithms (such as the ones described in $[21,28]$ ), the propagated phase map using the EM field carries reconstruction errors essentially at the edges of the embryo (see zoom in image 4.b). These errors arise from the intensity and phase noise which, combined with the high phase signal, generates local singularities and branch-cuts due to the phase wrapping. This artificial singularities can lead to phase errors when unwrapping is performed. Since the Rytov field conserves an unwrapped phase during the numerical propagation, it remains free from any singularity-based errors after the propagation. Here again the step-by-step refocusing leads to a better diffraction ring propagation and tends to be more accurate than the direct Rytov refocusing.

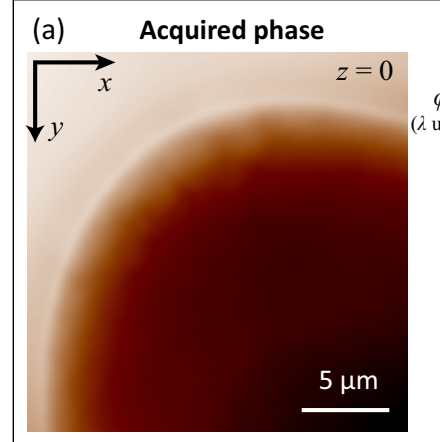

(c) Direct Rytov field num. propag.

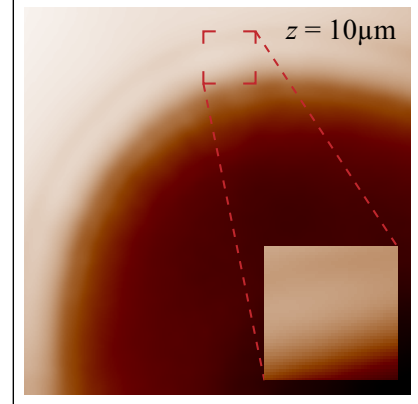

(b) EM field numerical propagation

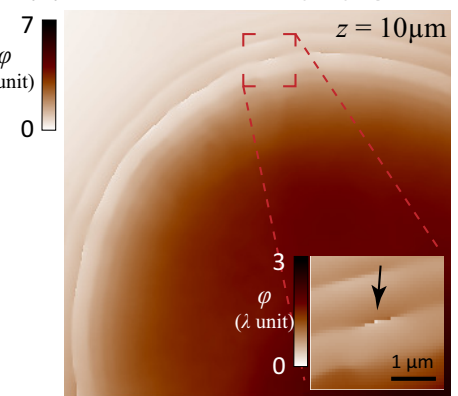

(d) Step-by Step Ryt. num. propag.

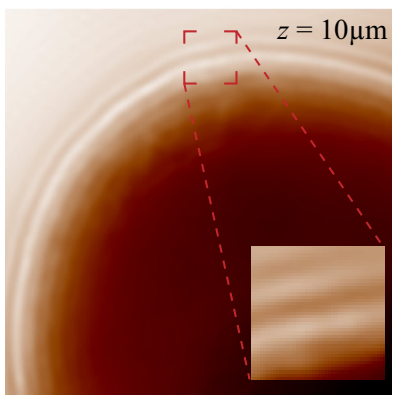

Fig. 4. Numerical propagation on a biological sample (drosophila embryo). (a) Experimental quantitative phase image of a drosophila embryo head. (b) Numerical defocusing using the EM field showing multiple phase singularity (see e.g. zoom, black arrow). (c) Same as (b) with a numerical defocusing using the Rytov field, no phase singularity (see zoom). (d) Same as (b) with a step-by-step numerical propagation using the Rytov field, no phase singularity and better accuracy on diffraction rings (see zoom).

\section{CONCLUSION}

We have demonstrated that numerical refocusing is possible using the first Rytov approximation of the scalar EM field. The validity of this approximation has been discussed and depends on the phase amplitude, spatial frequencies and refocusing distance. For regular biological samples, direct numerical refocusing at distance of 5 to 10 Rayleigh distances is possible with very limited errors. We have also introduced a step-by-step Rytov field defocusing algorithm to increase the reliability of the method especially for high-frequency phase information and upon large defocus. Numerical refocusing using the Rytov field is performed without phase-wrapping. This is of major interest especially when considering propagation of large phase values and/or when the phase noise is important which lead to artificial phase singularities, possibly phase-unwrapping algorithm failure and thus errors in refocused phase maps. The Ry- 
tov approximation is very useful when applied in combination with quantitative phase techniques that are intrinsically robust against phase-wrapping problems such as wavefront-sensing based approaches. Step-by-step Rytov propagation could also be considered and adapted in the scope of diffraction tomography.

\section{REFERENCES}

1. P. Langehanenberg, G. von Bally, and B. Kemper, "Autofocusing in digital holographic microscopy," 3D Res. 2, 4 (2011).

2. P. Bon, J. Savatier, M. Merlin, B. Wattellier, and S. Monneret, "Optical detection and measurement of living cell morphometric features with single-shot quantitative phase microscopy," J. Biomed. Opt. 17, 076004 (2012).

3. T. A. Zangle, M. A. Teitell, and J. Reed, "Live cell interferometry quantifies dynamics of biomass partitioning during cytokinesis," PloS one $\mathbf{9}$, e115726 (2014).

4. P. Bon, N. Bourg, S. Lécart, S. Monneret, E. Fort, J. Wenger, and S. Lévêque-Fort, "Three-dimensional nanometre localization of nanoparticles to enhance super-resolution microscopy," Nat. Commun. 6, 7764- (2015)

5. E. Cuche, P. Marquet, and C. Depeursinge, "Simultaneous amplitudecontrast and quantitative phase-contrast microscopy by numerical reconstruction of fresnel off-axis holograms," Appl. Opt. 38, 6994-7001 (1999).

6. G. Popescu, L. P. Deflores, J. C. Vaughan, K. Badizadegan, H. Iwai, R. R. Dasari, and M. S. Feld, "Fourier phase microscopy for investigation of biological structures and dynamics," Opt. Lett. 29, 2503-2505 (2004).

7. M. Debailleul, B. Simon, V. Georges, O. Haeberlé, and V. Lauer, "Holographic microcopy and diffractive microstomography of transparent samples," Meas. Sci. Technol. 19, 074009 - 074018 (2008).

8. S. Y. Suck, S. Collin, N. Bardou, Y. D. Wilde, and G. Tessier, "Imaging the three-dimensional scattering pattern of plasmonic nanodisk chains by digital heterodyne holography," Opt. Lett. 36, 849-851 (2011).

9. B. Kemper, A. Vollmer, C. E. Rommel, J. Schnekenburger, and G. von Bally, "Simplified approach for quantitative digital holographic phase contrast imaging of living cells," J. Biomed. Opt. 16, 026014 (2011).

10. B. Bhaduri, K. Tangella, and G. Popescu, "Fourier phase microscopy with white light," Biomed. Opt. Express 4, 1434-1441 (2013).

11. Z. Jingshan, L. Tian, J. Dauwels, and L. Waller, "Partially coherent phase imaging with simultaneous source recovery," Biomed. Opt. Express 6, 257-265 (2015).

12. C. Allier, S. Morel, R. Vincent, L. Ghenim, F. Navarro, M. Menneteau, T. Bordy, L. Hervé, O. Cioni, X. Gidrol et al., "Imaging of dense cell cultures by multiwavelength lens-free video microscopy," Cytom. Part A 91, 433-442 (2017).

13. I. Iglesias, "Pyramid phase microscopy," Opt. Lett. 36, 3636-3638 (2011).

14. P. Bon, G. Maucort, B. Wattellier, and S. Monneret, "Quadriwave lateral shearing interferometry for quantitative phase microscopy of living cells," Opt. Express 17, 13080-13094 (2009).

15. A. B. Parthasarathy, K. K. Chu, T. N. Ford, and J. Mertz, "Quantitative phase imaging using a partitioned detection aperture," Opt. Lett. 37, 4062-4064 (2012).

16. H. Gong, T. E. Agbana, P. Pozzi, O. Soloviev, M. Verhaegen, and G. Vdovin, "Optical path difference microscopy with a shack?hartmann wavefront sensor," Opt. Lett. (2017).

17. P. Berto, H. Rigneault, and M. Guillon, "Wavefront sensing with a thin diffuser," Opt. Lett. 42, 5117-5120 (2017).

18. M. Guillon and M. A. Lauterbach, "Quantitative confocal spiral phase contrast," J. Opt. Soc. Am. A 31, 1215-1225 (2014).

19. P. Bon, S. Lécart, E. Fort, and S. Lévêque-Fort, "Fast label-free cytoskeletal network imaging in living mammalian cells," Biophys. J. 106, 1588 - 1595 (2014).

20. J. F. Nye and M. V. Berry, "Dislocations in wave trains," Proc. Royal Soc. Lond. A: Math. Phys. Eng. Sci. 336, 165-190 (1974).

21. R. M. Goldstein, H. A. Zebker, and C. L. Werner, "Satellite radar interfer- ometry: Two-dimensional phase unwrapping," Radio Sci. 23, 713?720 (1988).

22. Y. Sung, W. Choi, C. Fang-Yen, K. Badizadegan, R. R. Dasari, and M. S. Feld, "Optical diffraction tomography for high resolution live cell imaging," Opt. Express 17, 266-277 (2009).

23. J. Lim, K. Lee, K. H. Jin, S. Shin, S. Lee, Y. Park, and J. C. Ye, "Comparative study of iterative reconstruction algorithms for missing cone problems in optical diffraction tomography," Opt. Express 23, 16933-16948 (2015).

24. S. S. Kou and C. J. Sheppard, "Imaging in digital holographic microscopy," Opt. Express 15, 13640-13648 (2007).

25. P. Bon, S. Aknoun, S. Monneret, and B. Wattellier, "Enhanced 3d spatial resolution in quantitative phase microscopy using spatially incoherent illumination," Opt. Express 22, 8654-8671 (2014).

26. P. Bon, B. Wattellier, and S. Monneret, "Modeling quantitative phase image formation under tilted illuminations," Opt. Lett. 37, 1718-1720 (2012).

27. A. J. Devaney, "Inverse-scattering theory within the rytov approximation," Opt. Lett. 6, 374-376 (1981).

28. D. Ghiglia and M. Pritt, Two-dimensional Phase unwrapping: Theory, Algorithms, and Software (Wiley, New York, 1998). 